\title{
Téoros
}

Revue de recherche en tourisme

\section{Technologie et destination}

Quelques initiatives visant à réduire la fracture numérique

\section{François Bédard et Boualem Kadri}

Volume 26, numéro 2, été 2007

URI : https://id.erudit.org/iderudit/1070951ar

DOI : https://doi.org/10.7202/1070951ar

Aller au sommaire du numéro

Éditeur(s)

Université du Québec à Montréal

ISSN

0712-8657 (imprimé)

1923-2705 (numérique)

Découvrir la revue

Citer cette note

Bédard, F. \& Kadri, B. (2007). Technologie et destination : quelques initiatives visant à réduire la fracture numérique. Téoros, 26(2), 77-80.

https://doi.org/10.7202/1070951ar d'utilisation que vous pouvez consulter en ligne.

https://apropos.erudit.org/fr/usagers/politique-dutilisation/ 


\section{Centre international de formation et de recherche en tourisme \\ Technologie et destination \\ Quelques initiatives visant à réduire la fracture numérique}

\section{François Bédard et Boualem Kadri}

Dans les pays en développement et tout particulièrement dans les pays les moins avancés, le secteur le plus important pour ce qui est de la croissance économique, des devises, des investissements et de la création d'emplois se révèle être presque partout le tourisme, ce qui démontre l'étroite corrélation entre tourisme et lutte contre la pauvreté. De fait, à condition d'être mis à profit comme il convient, les technologies de l'information et de la communication ( $\mathrm{TIC}$ ) et le tourisme sont des partenaires idéaux. Non seulement en théorie mais aussi dans la pratique, les TIC facilitent le développement des affaires et offrent aux entreprises et aux destinations, surtout des pays les moins avancés, un accès relativement facile et peu coûteux au marché mondial ${ }^{1}$.

L'accès effectif aux TIC est primordial pour le développement des pays les plus pauvres. Or, si celles-ci sont bien un instrument important pour atteindre les objectifs de la Déclaration du Millénaire de l'Organisation des Nations Unies adoptée en septembre $2000^{2}$, telle la réduction de la pauvreté, elles risquent, faute d'être exploitées correctement, d'élargir le fossé entre les économies développées et les économies en développement. Malgré de nombreux efforts allant dans le bon sens, force est de constater qu'il existe actuellement une fracture numérique entre ceux qui ont accès aux TIC et ceux qui en sont privés. II s'agit d'un défi planétaire que les Nations Unies veulent relever en priorité ; le Sommet mondial sur la société de l'information ${ }^{3}$ constitue une initiative tangible pour atteindre cet objectif.

Dans une précédente chronique (Bédard et Kadri, 2004), nous avons mis l'accent sur la problématique de la fracture numérique entre pays développés et pays en développement, notamment à travers la domination des voyagistes dans le système international touristique, engendrant un déséquilibre commercial entre les voyagistes du Nord et les destinations du Sud. La présente chronique illustre quelques initiatives d'organisations internationales visant à réduire cette fracture numérique. Une typologie des organisations a déjà été élaborée en ce qui concerne les programmes d'intervention de la période 1993-2000. Cette typologie montrait l'importance de l'OMT (Organisation mondiale du tourisme) et de la CNUCED (Conférence des Nations Unies sur le commerce et le développement) en tant qu'organisations de type global intervenant dans la "sensibilisation des acteurs économique et politique, avec un objectif, toutefois, de transformation des pratiques économiques par les nouvelles technologies » (Bédard et Kadri, 2003 : 41).

\section{Le concept de fracture numérique}

II convient ici de préciser ce qu'on entend par fracture numérique. Selon Bédard et Kadri (2003), le concept de fracture numérique renvoie tantôt à l'aspect technologique (matériel), tantôt à l'aspect informationnel. En fait, le concept ne peut se réduire à une seule dimension (économique, technologique ou sociale), mais présenterait plutôt deux pôles : le pôle matériel (les équipements technologiques) et celui de l'immatériel (l'information et les connaissances). En outre, la dimension immatérielle semble dominer de plus en plus aussi bien l'approche conceptuelle du phénomène que sa traduction en divers programmes et engagements pour enclencher le processus de réduction de la fracture numérique entre les pays développés et ceux en développement. Toujours selon ces auteurs, le concept de fracture numérique apparaît à la fois dans sa dimension statique (un écart à mesurer) et dans sa dimension dynamique. Le processus de réduction de la fracture numérique renferme sept composantes qui relèvent de la dimension dynamique du concept de fracture numérique et se définissent sur les plans de la technologie, de l'économie, du social, de la culture, de la science, du développement, de la société de l'information. Cette vision dynamique devient davantage significative lorsqu'elle est liée au processus au sein duquel s'inscrit le tourisme; ce dernier devient alors un élément fondamental des politiques et des stratégies des grandes organisations internationales, telles l'OMT et la CNUCED, engagées dans le processus de réduction de la fracture numérique entre le Nord et le Sud et dans la lutte contre la pauvreté.

\section{L'Organisation mondiale du tourisme}

Avec le concours d'autres institutions internationales et en collaboration avec ses propres États membres, I'OMT veille à aider ses membres dans leurs efforts de réduction de la fracture numérique. À cette fin, elle s'emploie à renforcer leur capacité de bénéficier des TIC et d'en améliorer l'utilisation dans le secteur du tourisme.

Dès 2002-2003, comme en fait état le programme de travail à sa section «Nouvelles technologies ", l'OMT s'est fixé comme objectif de jouer un rôle de leader en matière de technologies de l'information et de la communication appliquées au tourisme et de guider ses membres dans la réalisation de nouvelles initiatives dans ce domaine. 
L'Internet et les autres technologies de l'information sont en train de révolutionner la manière de faire des affaires dans l'industrie du tourisme, notamment la promotion des destinations. Le programme de travail de l'OMT en ce qui concerne les TIC vise à jouer un rôle de leader en la matière et à travailler à réduire la fracture numérique entre ses membres. L'OMT mène des études sur l'utilisation des TIC dans la promotion et le développement du tourisme. Elle diffuse les résultats de ces études au moyen d'une série de séminaires régionaux. Par ailleurs, elle anime un groupe d'experts des secteurs public et privé pour la conseiller. L'objectif est d'informer régulièrement ses membres sur les nouveaux développements technologiques et sur les impacts prévisibles pour l'industrie du tourisme.

Dans les lignes qui suivent, nous présentons brièvement, en nous référant à des communiqués de presse de l'OMT, quelques-unes de leurs initiatives en matière de TIC.

\section{Portail « Fenêtres ouvertes sur l'Afrique »}

En juillet 2006, l'Organisation mondiale du tourisme et la société Microsoft ont conclu un partenariat de longue durée pour l'expansion des technologies de l'information et de la communication dans le secteur touristique. Ce partenariat vise à fournir des systèmes et des instruments de TIC aux jeunes secteurs du tourisme afin d'améliorer leur compétitivité à l'exportation, la qualité de leurs produits et leurs compétences logistiques. Sa cible principale est l'Afrique. Dans son discours-programme au Forum mondial des leaders pour l'Afrique, Bill Gates, président de Microsoft et spécialiste de l'architecture logicielle, déclarait que le partenariat publicprivé avec l'OMT était le genre de programme d'application qui aidera plus de 40 millions d'Africains à avancer vers un avenir de croissance économique. Microsoft veut jouer un rôle direct pour aider des régions et des pays entiers à développer une économie fondée sur le savoir, à créer des emplois, à aiguillonner la croissance et à encourager l'innovation. II estime pouvoir le faire en assurant un accès facile et bon marché à la technologie et en permettant aux partenaires de l'OMT de bâtir de solides économies du logiciel à l'échelle locale.

Parmi les premiers projets découlant de la collaboration avec Microsoft, mentionnons celui de la création d'un portail appelé «Fenêtres ouvertes sur l'Afrique ", grâce à la coopération entre l'OMT et le NEPAD (New Partnership for Africa's Development). Ce portail vise à présenter une Afrique compétitive sur le plan mondial et il pourra être reproduit aux échelons national et local. II comportera des liens vers des centres pour visiteurs, des musées et des bibliothèques. Par l'intermédiaire des assistants numériques personnels (PDA) et des appareils portables, il finira même par fournir des conseils en temps réel et des données sur des destinations particulières.

Cet accord de partenariat lie les solutions technologiques de Microsoft au rôle essentiel de l'OMT qui consiste à promouvoir le secteur comme instrument de développement. Au cours des cinq prochaines années, les deux partenaires collaboreront étroitement pour donner à tous les grands programmes de l'OMT une dimension à la pointe des TIC - de l'amélioration de l'accès pour les touristes avec le contrôle électronique aux frontières à des services de meilleure qualité, en passant par la formation à distance et la gestion de la durabilité dans les destinations.

\section{Réseau social de cybertourisme}

En février 2007, l'OMT et WISeKey (compagnie spécialisée dans les systèmes de sécurité pour Internet) ont annoncé un accord visant à développer l'utilisation des techniques d'identification et d'authentification des voyageurs dans le secteur du tourisme. Cette collaboration s'ajoute à l'accord de partenariat public-privé que l'OMT et Microsoft ont signé en 2006. Les nouveaux partenaires ont pour but de mettre sur pied un réseau social de cybertourisme unique en son genre: YouTourist. Exploitant les techniques d'identification numérique de WISeKey et Cardspace ${ }^{4}$ de Microsoft, cette plate-forme permettra aux touristes de se relier les uns aux autres et d'échanger de l'information sur la base du Code mondial d'éthique du tourisme de l'OMT. Le Code est un instrument pour sauvegarder les ressources dont dépend le tourisme, en veillant à ce que ses avantages soient répartis équitablement, conformément aux objectifs du Millénaire pour le développement. II énonce des principes directeurs pour les gouvernements, les destinations, les voyagistes, les agents de voyages, les travailleurs du secteur, les promoteurs et les voyageurs eux-mêmes.

L'explosion des réseaux sociaux correspond parfaitement à l'esprit du tourisme responsable et durable que l'OMT cherche à encourager. YouTourist favorisera, entre les touristes, les échanges de photos, de vidéodisques et de commentaires et offrira des possibilités spéciales de communication aux communautés et aux entreprises locales qui interviennent dans toute la chaîne de développement du tourisme. L'adoption du type de techniques disponibles pour les réseaux sociaux sécurisés du secteur touristique peut être d'une grande aide dans les actions transversales pour réduire la pauvreté. L'identification numérique servant à protéger les participants sera un élément clé.

\section{Gestion des données et de la sécurité électronique}

En avril 2007, I'OMT signait un accord de collaboration avec Indusa Global, spécialiste de la gestion des données et de la sécurité électronique, afin de faire progresser l'application des techniques de l'information et de la communication dans l'intérêt du développement durable du secteur du tourisme. Cet accord s'inscrit dans le partenariat public-privé de longue durée entre l'OMT et Microsoft, qui a pour résultats une série de projets qui aident à offrir aux touristes des expériences caractérisées par la sécurité et la qualité. II comportera une collaboration étroite avec WISeKey, autre partenaire de l'OMT, et Microsoft dans le domaine de l'eTD (tourisme électronique au service du développement), pour des projets comme la stratégie SAFE d'amélioration de la sécurité et de la facilitation, le portail "Fenêtres ouvertes sur l'Afrique ", le système d'intervention d'urgence [www.sos.travel] et le réseau social pour les voyageurs responsables [www. YouTourist.net].

Les efforts porteront en priorité sur le passage des frontières et, en particulier, sur les visas électroniques et veillera surtout à ce que les pays les plus pauvres du monde soient en mesure de participer à ce genre de programmes. Cet accord renforce l'engagement de I'OMT de créer un environnement de voyage sécurisant et agréable pour tous les touristes, autrement dit d'accroître la sécurité tout en facilitant les voyages. 


\section{Centre mondial d'excellence des destinations (CED)}

L'OMT a apporté son appui à la mise en place du Centre mondial d'Excellence des Destinations, une initiative lancée par deux de ses membres affiliés, Tourisme Montréal et l'Université du Québec à Montréal (UQAM). Outre les deux initiateurs du CED, d'autres organismes comptent parmi ses membres fondateurs, notamment Bell Canada. Les problématiques liées aux TIC figurant parmi les priorités de ce nouveau centre, il est apparu opportun de faire un lien avec le projet "E-tourisme pour le développement », fruit d'un partenariat entre I'UQAM et Bell Canada. Ce partenariat propose un programme de recherche qui s'inscrit dans un contexte mondial où le tourisme est de plus en plus reconnu par les grandes agences intergouvernementales et bilatérales comme un facteur important pour le développement et la réduction de la pauvreté. Le programme de recherche s'articule autour d'un certain nombre d'axes, notamment les problématiques liées aux critères de succès d'un système de gestion de destination, à la gestion de la relation clients dans les organismes de gestion de destination, au comportement des internautes dans leur utilisation de sites Web de destinations touristiques, à la réduction de la fracture numérique entre les pays développés et les pays en voie de développement ou les moins développés, au financement de projets en e-tourisme dans le contexte de la coopération internationale.

\section{Conférence des Nations Unies sur le commerce et le développement}

Le tourisme représente un des plus importants secteurs d'exportation pour les 50 pays les moins avancés (PMA). Cependant, en parallèle, les fuites (par exemple, rapatriement de profits) dans le secteur du tourisme peuvent atteindre $40 \%$ en Inde, $70 \%$ en Thaillande, $80 \%$ dans les Carailbes et jusqu'à $85 \%$ dans certains PMA africains. Afin de minimiser ces fuites, de promouvoir une croissance durable et de contribuer à la réduction de la pauvreté, il est maintenant essentiel pour les destinations touristiques d'accroître leur autonomie et d'intégrer toutes les petites entreprises du secteur habituellement exclues de l'offre. Dans le but de relever ce défi et ainsi d'aider les pays en développement, et tout particulièrement les PMA, à exploiter au mieux leur potentiel touristique, la CNUCED a développé I'Initiative e-tourisme ${ }^{5}$.

\section{L'Initiative e-tourisme}

Lancée lors de la onzième conférence ministérielle quadriennale de la CNUCED, qui s'est tenue à São Paulo, au Brésil, en juin 2004, I'Initiative e-tourisme fait partie du Groupe de travail sur le tourisme durable pour le développement. Articulant tourisme durable et TIC pour le développement, cette initiative, basée sur la demande, va renforcer les capacités des pays en développement de promouvoir leur propre offre touristique et faciliter son positionnement et son adaptation dynamique sur le marché mondial. Ce projet se focalise sur le développement et l'implantation d'outils basés sur les TIC, outils qui vont aider les communautés à mieux atteindre le marché international, en promouvant le développement et la participation de tous les acteurs du secteur touristique et l'appropriation locale des différents systèmes, ainsi qu'en renforçant les capacités humaines et institutionnelles nécessaires.

L'Initiative e-tourisme fait partie des activités de coopération technique de la CNUCED.
Pour beaucoup de pays en développement, et particulièrement pour les PMA, les petits États et les États insulaires en développement, le tourisme représente un potentiel significatif en matière de croissance durable, de réduction de la pauvreté et de développement. II constitue souvent la source majeure de devises étrangères et génère un nombre important d'emplois.

Les TIC contribuent à rendre les pays en développement plus autonomes et autosuffisants dans la construction de leur propre image de marque et la promotion de leurs atouts touristiques. En maximisant leurs avantages comparatifs dans ce secteur, les pays en développement peuvent ajuster leur offre touristique, en conformité avec leur stratégie de développement, et être mieux intégrés dans l'économie mondiale.

Les bénéficiaires de ce projet sont les ministères du tourisme, les offices nationaux du tourisme, les organisations de gestion des destinations, les agents touristiques locaux ainsi que d'autres partenaires pertinents du secteur.

L'objectif immédiat est de concevoir, de créer et d'établir une plate-forme pérenne de commerce électronique dans le secteur du tourisme, identifiant, promouvant et intégrant les fournisseurs locaux de biens et services, en tenant compte du contexte international. Parmi les autres objectifs, notons : permettre aux destinations d'organiser leur offre touristique, de développer de nouveaux produits, d'améliorer la qualité de leurs services ou d'encourager le développement de partenariats publics-privés afin d'améliorer la compétitivité du secteur.

L'Initiative e-tourisme s'articule autour d'un outil, d'une méthode et de la constitution d'alliances et de partenariats. L'outil, ou plate-forme électronique, vise à contribuer au recensement, à la standardisation, à la fédération et à la présentation de l'offre touristique nationale pour la confronter dynamiquement à la demande mondiale. Au-delà de la mise en place de la plate-forme technique, c'est aussi une méthode pour assurer la cueillette des données sur ces secteurs (tourisme et artisanat), leur standardisation et les mécanismes de leur dissémination sur Internet. L'objectif de cette méthode est d'encourager une coopération active entre les partenaires, de renforcer les capacités afin de promouvoir un développement durable. La mise en place de partenariats est donc très pertinente puisqu'elle permet d'harmoniser ressources et objectifs pour tous les acteurs, qu'ils soient privés ou publics. Les partenariats peuvent être formés dans le secteur privé, dans le secteur public, ou de manière trans-sectorielle.

Trois considérations particulières appuient la spécificité de la CNUCED dans le secteur du tourisme. II s'agit d'un soutien spécifique aux PME locales; d'une approche décentralisée et inclusive, grâce à la participation d'ONG (organisations non gouvernementales) qualifiées dans ce domaine; d'une action de conseil pertinente, liée à de l'assistance technique spécifique, afin de promouvoir et d'organiser l'offre touristique locale, par l'utilisation d'outils basés sur les TIC. La mise en oeuvre de l'Initiative e-tourisme fait partie des activités d'assistance technique de la CNUCED, activités liées à des procédures spécifiques. 
Le leitmotiv de l'initiative peut se résumer par la déclaration suivante de Rubens Ricupero, secrétaire général de la CNUCED (de septembre 1995 à septembre 2004) : "Si le secteur du tourisme est convenablement intégré dans des stratégies de développement, il peut constituer la pierre angulaire de nouveaux systèmes productifs permettant la réduction de la pauvreté et une meilleure intégration de ces pays dans l'économie mondiale. »6

\section{Partenariat entre la CNUCED et l'Université du Québec à Montréal}

Dans la foulée de l'Initiative e-tourisme, la CNUCED et I'UQAM ont signé à l'automne 2006 une entente de partenariat. Aux termes de cette entente, les deux partenaires peuvent, à l'intérieur de leur programmation respective, convenir de l'élaboration et de la réalisation d'activités conjointes et/ou complémentaires dans des projets en e-tourisme dans les pays en développement, notamment par : a) un appui technologique au déploiement progressif de systèmes de gestion de destination par les diverses contributions ou transferts technologiques nécessaires au développement et à la mise en œuvre locale de la plate-forme en logiciel libre à code ouvert ; b) un appui à la conception et à la mise en place de modèles d'affaires pour le tourisme électronique permettant aux intervenants touristiques de collaborer à la gestion de leur destination autour de la plate-forme e-tourisme; c) le transfert technologique correspondant aux ingénieurs issus des pays en développement qui ont décidé d'adopter la plate-forme, d'en maîtriser la technologie et de réaliser eux-mêmes les extensions souhaitées ; d) des contributions à la conception et à l'enseignement de modules de formations ad hoc pour les pays en développement bénéficiant de l'assistance technique de la CNUCED en matière d'e-tourisme ; e) l'accueil et le déroulement de stages d'étudiants.

\section{Conclusion}

L'OMT et la CNUCED, deux agences de la famille de l'Organisation des Nations Unies, se sont résolument engagées, au cours des dernières années, dans la réduction de la fracture numérique. Comme il vient d'être démontré, chacune de ces deux grandes organisations internationales ont lancé des initiatives qui sont le reflet du caractère distinctif de leur mission et de leur culture organisationnelle respective. Les initiatives de l'OMT portent principalement sur le développement de partenariats avec des fournisseurs privés de technologies de pointe dans le but d'en faciliter l'accès et leur utilisation par ses pays membres, notamment ceux des pays en développement d'Afrique. Quant à la CNUCED, son Initiative e-tourisme s'inscrit dans une démarche d'appropriation des TIC par ses pays membres, en donnant la priorité aux pays les moins avancés. La démarche d'appropriation débute par un accompagnement des différents intervenants publics et privés d'un pays dans l'élaboration d'une stratégie nationale en e-tourisme à partir de laquelle sera mis en place un système de gestion de la destination opérant sur une plate-forme en logiciel libre à code ouvert. L'UQAM, par son Centre international de formation et de recherche en tourisme (CIFORT), apporte son concours à ces différentes initiatives.
François Bédard est directeur du CIFORT et professeur au département d'études urbaines et touristiques de l'Université du Québec à Montréal.

Boualem Kadri, est professeur associé au département d'études urbaines et touristiques de l'Université du Québec à Montréal.

\section{Notes}

1 Extraits de la préface de Francesco Frangialli, secrétaire général de l'Organisation mondiale du tourisme (Bédard et Kadri, 2003).

2 [http://www.droitshumains.org/mill/decl_millen.htm], (consulté le 30 avril 2007). 54e session - Assemblée du Millénaire de l'Assemblée générale des Nations Unies, 6-8 septembre 2000, New York.

3 Le premier volet du sommet s'est tenu à Genève en 2003 et le second à Tunis en 2005. [http://www.itu.int/wsis/index-fr.html], (consulté le 30 avril 2007).

4 Cardspace est le nom d'un produit Windows qui permet la gestion de différentes identités numériques.

5 [http://www.unctadxi.org/templates/Startpage____1195.aspx], (consulté le 30 avril 2007). Initiative en e-tourisme de la CNUCED.

6 Idem.

\section{Bibliographie}

Bédard, François, et Boualem Kadri (2003), Réduction de la fracture numérique. Le rôle des grandes organisations internationales, Québec, Presses de l'Université du Québec.

Bédard, François, et Boualem Kadri (2004), «Les technologies de l'information comme facteur d'autonomie des destinations touristiques du Sud", Téoros, vol. 23, n² 2 .

Bédard, François, Boualem Kadri, et Michèle Laliberté (2002), "Systèmes de gestion de destination dans les pays en développement ", Revue TEDQUAL, n 5, 1/2002, Organisation mondiale du tourisme.

CNUCED (2005), «Les perspectives offertes par le tourisme électronique dans les pays en développement ", chap. sur "Le commerce électronique et le développement, rapport de la CNUCED sur l'économie de l'information ", Genève, CNUCED.

OMT (1999), La commercialisation en ligne des destinations touristiques, Madrid, OMT.

OMT (2001), Commerce électronique et tourisme : Guide pratique pour les destinations et les entreprises, Madrid, OMT.

OMT (2005), Évaluation et amélioration des sites Web, le Système de veille électronique des sites des destinations, Madrid, OMT. 